\title{
Do Vigorous Esophageal Contractions Contribute to Rigorous Phenotyping of Gastroesophageal Reflux Disease?
}

\author{
Rena Yadlapati ${ }^{1,2} \cdot$ Samir Gupta ${ }^{1,3} \cdot$ John E. Pandolfino ${ }^{4}$ \\ Published online: 4 July 2019 \\ ○) Springer Science+Business Media, LLC, part of Springer Nature 2019
}

Keywords Ineffective esophageal motility $\cdot$ Manometry $\cdot$ Personalized management

\author{
Abbreviations \\ GERD Gastroesophageal reflux disease \\ IEM Ineffective esophageal motility
}

Nearly one third of the adult US population experiences troublesome symptoms such as heartburn or regurgitation suggestive of gastroesophageal reflux disease (GERD). Historically, empirical management strategies for GERD have resulted in suboptimal patient outcomes and a substantial healthcare burden [1]. With the increased recognition that patients with GERD are heterogeneous, the management of GERD is now shifting toward phenotype-guided treatment. Conceptual frameworks to phenotype GERD take into consideration the integrity of the anti-reflux barrier, the pattern of reflux burden, the mechanism of gastroesophageal reflux events, visceral sensitivity, and effectors of esophageal clearance $[2,3]$. The latter, effectors of esophageal clearance, is primarily measured by the vigor of esophageal contractility as assessed by high-resolution esophageal manometry. When the vigor of at least half of test swallows on manometry is weak (distal contractile integral less than $450 \mathrm{mmHg} \mathrm{s} \mathrm{cm}$ ), ineffective esophageal motility (IEM) is diagnosed according to the Chicago classification version 3.0 [4]. Although IEM is the most commonly encountered motility disorder

Rena Yadlapati

renahyadlapati@gmail.com

1 Division of Gastroenterology and Hepatology, School of Medicine, University of California San Diego, San Diego, CA, USA

2 Division of Gastroenterology and Hepatology, University of Colorado Anschutz Medical Campus, Academic Office 1 Room 7605, 12631 E. 17th Ave, Aurora, CO 80045, USA

3 VA San Diego Healthcare System, San Diego, CA, USA

4 Division of Gastroenterology and Hepatology, Northwestern University Feinberg School of Medicine, Chicago, IL, USA diagnosed with manometry, frequently found in asymptomatic patients and in patients with GERD, the clinical relevance of identifying IEM in order to phenotype and manage GERD remains unclear.

In this issue of Digestive Diseases and Sciences, Reddy and colleagues aim to characterize gastroesophageal reflux patterns among patients with IEM [5]. This retrospective cross-sectional study of esophageal manometry studies comparing up to 239 patients with IEM and 100 patients with normal esophageal motor function reports that patients with IEM have a higher number of reflux events as measured with impedance-pH monitoring (on or off acid suppression) and a greater acid exposure time as measured with $\mathrm{pH}$ monitoring performed off acid suppressive therapy. Furthermore, patients with IEM have a lower esophagogastric junction contractile integral, a surrogate measure of anti-reflux barrier integrity [6]. The authors further sub-group patients with IEM according to esophagogastric junction morphology type (I, II, and III), and note that patients with IEM and esophagogastric junction morphology type III $(>2 \mathrm{~cm}$ separation between the crural diaphragm and lower esophageal sphincter) have a higher body mass index, greater acid exposure, higher number of reflux events, and higher bolus exposure time.

The findings by Reddy and colleagues corroborate the notion that IEM is associated with GERD-associated pathologies such as increased reflux burden, a disrupted anti-reflux barrier, and obesity. An issue that remains unresolved in this observational study is the directionality of association. Does reduced esophageal contractility impair clearance of refluxate, increasing gastroesophageal reflux burden, or does chronic gastroesophageal reflux lead to esophageal injury and dysfunctional esophageal peristalsis? Regardless of the underlying pathophysiology, esophageal contractility seems to be a physiologically relevant component of GERD.

As the authors mention, the clinical significance of IEM in GERD is unknown. Thus, inclusion of patient-reported 
outcome instruments such as the GerdQ and the Brief Esophageal Dysphagia Questionnaire to measure the burden of symptoms in patients with IEM is a clear strength of the study design. Nevertheless, the authors do not identify any major differences in symptom burden in patients with IEM compared to those with normal esophageal motor function, possibly since the study may have been underpowered to detect meaningful differences. Moreover, symptom perception is multi-modal and influenced by unmeasured factors such as esophageal hypersensitivity and hypervigilance [7]. Therefore, this negative finding provokes the question: do patients with GERD require different management on the basis of esophageal motor function?

This work also heralds a growing challenge with regards to design of studies aiming to characterize phenotypes of GERD, and explore potential causal mechanisms. Since this study compared a sample of patients undergoing routine manometry with IEM to a random sample of individuals without IEM, it is not well suited to explore the relative prevalence of IEM among patients with a symptom complex of interest (in this case GERD), and the correlation or interaction of the key predictors of interest (presence vs. absence of IEM) with other parameters that could contribute to the symptom complex. Future studies should consider more rigorous observational designs, such as case-control and longitudinal cohort studies. For example, a case-control study could identify a study base of patients undergoing manometry, select a random sample of cases with the phenotype of interest (GERD on reflux monitoring) and a sample of controls without the phenotype, and be in a stronger position to understand the independent association between a key predictor such as IEM and case status. Further, this proposed study design could consider potential interactions and correlations between IEM and other factors and GERD risk, as well as examine its prevalence relative to other physiologic parameters and attributable risk.

Regardless of the physiologic or clinical relevance of IEM in GERD, and pending more rigorous studies, wellestablished management strategies used to treat IEM are not available. Reddy and colleagues suggest that the increased reflux events in patients with IEM may reflect an increased burden of transient lower esophageal sphincter relaxations in IEM. Therefore, inhibition of transient lower esophageal sphincter relaxations with GABA agonists is a potentially effective treatment strategy in patients with GERD and IEM. Accordingly, future work assessing the frequency of transient lower esophageal sphincter relaxations in GERD with IEM will be of interest. At present, since we speculate that the increased reflux events in IEM are related to a hypotensive lower esophageal sphincter and impaired anti-reflux integrity, adjusting for covariates in this statistical model may have been able to identify these interactions. Another shortcoming of the statistical model is the categorical nature of the primary outcome, which is dichotomized as normal esophageal motor function or IEM. For instance, the defined outcome categorizes a patient with $0 \%$ or $40 \%$ of weak swallows as normal, and a patient with $50 \%$ or $90 \%$ weak swallows as IEM even though all four represent different points on the spectrum of esophageal vigor, and those with $40 \%$ or $50 \%$ weak swallows are more similar than distinct.

Although the clinical utility of IEM in phenotyping patients with GERD is not clear, esophageal vigor and peristaltic reserve are relevant in terms of outcomes following anti-reflux surgery. Poor esophageal peristaltic reserve has been associated with late postoperative dysphagia and therefore may limit a patient's candidacy or require a tailored anti-reflux procedure [8-10]. Thus, characterizing esophageal motor function in GERD is an important component in the preoperative evaluation of foregut altering procedures.

In summary, Reddy and colleagues characterize patterns of reflux burden among patients with IEM, building on the existing literature in order to highlight the interrelation between esophageal hypomotility and gastroesophageal reflux burden. Moreover, this study highlights important gaps in knowledge as the field shifts toward phenotypeguided management for GERD and the need for more rigorous study designs in order to understand the relationship of measurable esophageal physiologic parameters and GERD. In order to translate the compelling physiologic findings from this study to the clinical setting, adequately powered outcomes studies need to examine treatment strategies according to GERD phenotype, taking into account a measure of esophageal contractility. Implicit to this is, further, the need for therapies that will effectively address esophageal contractility. This work underscores the clinical relevance of esophageal contractility in phenotyping and personalizing GERD management.

Acknowledgments RY \& JEP are supported by NIH R01 DK092217 (JEP); RY is supported by American College of Gastroenterology Junior Faculty Development Award.

Author's contribution RY, SG, and JEP analyzed and provided an interpretation of the paper, conducted a literature review, drafted the initial manuscript, critically revised the manuscript for important intellectual content, finalized the manuscript, and participated in revising the manuscript and addressing the reviewers' comments.

\section{Compliance with Ethical Standards}

Conflict of interest RY: Consultant for Ironwood, Medtronic, Diversatek; JEP: Consultant for Crospon, Ironwood, Torax, Astra Zeneca, Takeda, Impleo, Medtronic, Sandhill. 


\section{References}

1. El-Serag HB, Sweet S, Winchester CC, Dent J. Update on the epidemiology of gastro-oesophageal reflux disease: a systematic review. Gut. 2014;63:871-880.

2. Gyawali CP, Roman S, Bredenoord AJ, Fox M, Keller J, Pandolfino JE, et al. Classification of esophageal motor findings in gastro-esophageal reflux disease: conclusions from an international consensus group. Neurogastroenterol Motil. 2017. https:// doi.org/10.1111/nmo.13104.

3. Yadlapati R, DeLay K. Proton pump inhibitor-refractory gastroesophageal reflux disease. Med Clin North Am. 2019;103:15-27.

4. Kahrilas PJ, Bredenoord AJ, Fox M, et al. The Chicago Classification of esophageal motility disorders, v3.0. Neurogastroenterol Motil. 2015;27:160-174.

5. Reddy CA, Baker JR, Lau J, Chen JW. High-resolution manometry diagnosis of ineffective esophageal motility is associated with higher reflux burden. Dig Dis Sci. (Epub ahead of print). https:// doi.org/10.1007/s10620-019-05633-3.

6. Nicodeme F, Pipa-Muniz M, Khanna K, Kahrilas PJ, Pandolfino JE. Quantifying esophagogastric junction contractility with a novel HRM topographic metric, the EGJ-Contractile Integral: normative values and preliminary evaluation in PPI non-responders. Neurogastroenterol Motil. 2014;26:353-360.

7. Aziz Q, Fass R, Gyawali CP, Miwa H, Pandolfino JE, Zerbib F. Functional esophageal disorders. Gastroenterology. 2016. https:// doi.org/10.1053/j.gastro.2016.02.012.

8. Mello MD, Shriver AR, Li Y, Patel A, Gyawali CP. Ineffective esophageal motility phenotypes following fundoplication in gastroesophageal reflux disease. Neurogastroenterol Motil. 2016;28:292-298.

9. Shaker A, Stoikes N, Drapekin J, Kushnir V, Brunt LM, Gyawali CP. Multiple rapid swallow responses during esophageal highresolution manometry reflect esophageal body peristaltic reserve. Am J Gastroenterol. 2013;108:1706-1712.

10. Yadlapati R, Hungness ES, Pandolfino JE. Complications of antireflux surgery. Am J Gastroenterol. 2018;113:1137-1147.

Publisher's Note Springer Nature remains neutral with regard to jurisdictional claims in published maps and institutional affiliations. 